\title{
Development of Leaky-Wave Antenna Applications with Acoustics Metamaterials: from the Acoustic Dispersive Prism to Sound Direction Finding with a Single Microphone
}

\author{
$\underline{\text { H. Lissek }}^{1}$, H. Esfahlani ${ }^{1}$, J.R. Mosig ${ }^{2}$ and S. Karkar ${ }^{3}$ \\ ${ }^{1}$ Ecole Polytechnique Fédérale de Lausanne, Laboratoire de Traitement des Signaux LTS2, EPFL STI IEL \\ LTS2, Station 11, CH-1015, Lausanne, Switzerland \\ ${ }^{2}$ Ecole Polytechnique Fédérale de Lausanne, Laboratoire d'Electromagnétisme et Antennes LEMA, EPFL \\ STI IEL LEMA, Station 11, CH-1015, Lausanne, Switzerland \\ ${ }^{3}$ Ecole Centrale de Lyon, Laboratoire de Tribologie et Dynamique des Systèmes LTDS, 36 avenue Guy de \\ Collongue, F-69134 Ecully, France \\ herve.lissek@epfl.ch
}

\begin{abstract}
Recent studies have focused on developing metamaterials for acoustic applications, inspired by electromagnetics concepts. The acoustic leaky-wave antenna is amongst the most investigated. Despite the unfavourable properties of conventional matter and structures with respect to sound dispersion and radiation, interesting engineering processes have been recently proposed that are likely to allow such peculiar properties. After presenting the developed onedimensional leaky-wave antenna design, this paper discusses two pioneering applications of the latter: the Acoustic Dispersive Prism and the Single-Microphone Direction Finding.
\end{abstract}

\section{INTRODUCTION}

More than 30 years after the first idea of an electromagnetic medium with simultaneous negative permittivity and permeability [1], an acoustic counterpart was mathematically reported by Li et al [2]. The authors suggested the existence of media with simultaneous negative mass density and bulk modulus, using impedance contrast between water and soft rubber spheres. Then, it took only a few years to see the first engineered structure exhibiting double negative properties on a broad frequency range [3]. The proposed structure was composed of a periodic arrangement of unit-cells of length $d$ made of a cylindrical duct, comprising an elastic membrane along its cross section, playing the role of the negative mass, and a transversal thin axisymmetric slits open to the outside, playing the role of negative bulk modulus. Such negative acoustic elements coexisting with "natural" positive constituents, the structure behaves as a Composite Right-/Left-Handed (CRLH) Acoustic Metamaterial, presenting adjustable dispersive medium properties. Such a structure was then shown to propagate sound waves with monoticallyvarying phase velocities depending on frequency. An alternative design was then proposed by Esfahlani et al. [4], having a plane symmetric design. In such a design, small cylindric ducts were substituted to the axisymmetric slits, allowing a better sound radiation. In the same time, a dedicated mounting system was proposed to assemble the Kapton membranes inside the host waveguide, so that their mechanical impedance alignment can be ensured. Thanks to this innovative design, the first Acoustic Dispersive Prism (ADP) has been achieved [5], together with the dual concept of Single-Microphone Direction Finding (SMDF) [6]. In this paper we remind the proposed LWA design, and then discuss the application to ADP and SMDF, with simulation and experimental results of the latter.

\section{Acoustic LeAKY-WaVe Antenna Design}

The proposed acoustic LWA is made by stacking together 10 unit-cells, each consisting of a portion of length $d$ of a cylindric tube (host waveguide) with radius $r_{\mathrm{w}}$, comprising an elastic Kapton membrane of thickness $t_{m}$, and connected to the outside through a side open cylindric tube of length $L_{\mathrm{s}}$ and radius $r_{\mathrm{s}}$ (the unit-cell is highlighted in red in Figure 1 top). Specific dimensions are assigned to the first and last portions of the host waveguide (length $b$ ) and side channels radius $\left(r_{\mathrm{s}, \mathrm{i} / \mathrm{o}}\right)$ to allow a good matching with input and output waveguides (radius $r_{\mathrm{w}, \mathrm{i} / \mathrm{o}}$ ) where either a loudspeaker or a microphone are inserted (depending on the application further presented in section III.). The dimensions are given in Table 1 . 


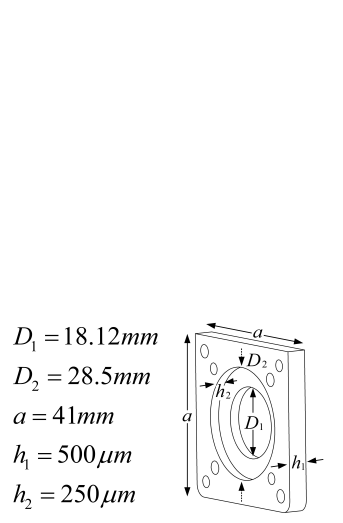

a)
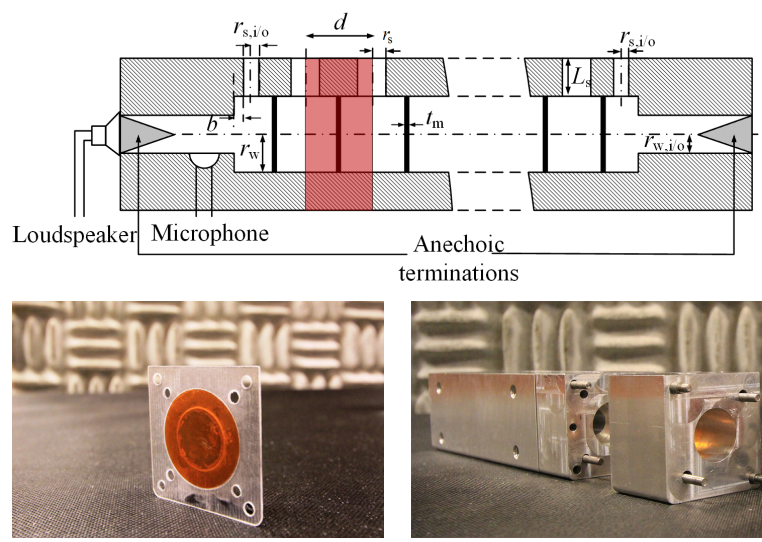

b)

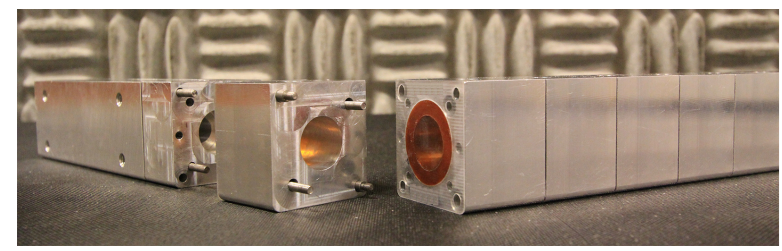

c)

Fig. 1: Top Description of the LWA design. The unit-cell is highlited in red; Bottom: a) Detailed design of the membranes support; b) Picture of the prototype membrane element; and c) assembly of the LWA prototype.

\begin{tabular}{lcc}
\hline Dimension & Symbol & Value $(\mathbf{m m})$ \\
\hline Unit-cell length & $d$ & 32.00 \\
Duct radius & $r_{\mathrm{w}}$ & 9.06 \\
Terminating ducts radius & $r_{\mathrm{w}, \mathrm{i} / \mathrm{o}}$ & 5.54 \\
Stub radius & $r_{\mathrm{s}}$ & 4.00 \\
Terminating stubs radius & $r_{\mathrm{s}, \mathrm{i} / \mathrm{o}}$ & 2.90 \\
Stub length & $L_{\mathrm{s}}$ & 12.50 \\
First stub position & $b$ & 1.10 \\
Membrane thickness & $t_{\mathrm{m}}$ & 0.125 \\
\hline
\end{tabular}

\begin{tabular}{lccc}
\hline Physical quantity & Symbol & Value & Unit \\
\hline Air mass density & $\rho$ & 1.188 & $\mathrm{~kg} / \mathrm{m}^{3}$ \\
Air Bulk modulus & $K$ & 137.4 & $\mathrm{kPa}$ \\
Celerity of sound in the air & $c$ & 340 & $\mathrm{~m} / \mathrm{s}$ \\
Kapton Youngs modulus & $E$ & 2.758 & $\mathrm{GPa}$ \\
Kapton Poisson ratio & $\nu$ & 0.34 & $(-)$ \\
Kapton mass density & $\rho_{m}$ & 1420 & $\mathrm{~kg} / \mathrm{m}^{3}$ \\
\hline
\end{tabular}

Table 1: LWA design dimension and physical properties

Such periodic structure has been shown to behave as a CRLH Acoustic Metamaterial [3], presenting monoticallyincreasing Bloch constant $\gamma_{B}(f)$, from negative to positive values, with a transition frequency $f_{0}=1 \mathrm{kHz}$. However, any misalignment of membranes impedances has been proven to significantly penalize the transmissibility of the transmission-line, thus reducing the interest for an application to LWA. In this design, the frame supporting the membranes, and the specific mounting setup described in Figure 1a), allow ensuring such a tuning, thus improving the transmission of sound power within the main waveguide. This dispersive medium characteristics allows varying phase velocities inside the host waveguide and frequency: below $1 \mathrm{kHz}$ with negative phase velocities, at $f=1$ $\mathrm{kHz}$ with infinite phase velocity, and above $1 \mathrm{kHz}$ with positive phase velocities.

Therefore, it is now possible to envisage the application of such a dispersive medium as Acoustic LWA, provided a good enough matching is ensured between the leaking components (here the transversal cylindric open channels) and the outside medium. This is achieved experimentally with the prototype presented in Figure 1, in two opposite applications: the ADP and the SMDF, presented hereafter.

\section{ApPlicAtions}

In both applications, the Acoustic LWA prototype is flush-mounted on a rigid screen, the side open channels on top, to improve the sensitivity in the half-space facing the openings.

\section{A. Acoustic Dispersive Prism}

In this application, a loudspeaker is mounted at one termination of the host waveguide, producing a broadband noise inside the structure. Thanks to the good transmission ensured by the framed membranes, and the good impedance matching with the outside medium ensured by the cylindric tubes, the sound waves can leak out, with 
varying time delays depending on frequency, owing to the dispersive nature of the host medium. Then, the structure can split broadband sound waves into its frequency constituents at various angles, thus behaving as an ADP.

\section{B. Single-Microphone Direction Finding}

In this application, a microphone is flush mounted in the input waveguide, and a loudspeaker, located 3 meters away from the screen supporting the LWA and directed towards angle $\theta$ with respect to the normal of the screen, generates broadband noise. The sound building up inside the host waveguide depends on the direction of arrival $\theta$. The structure behaves as a direction-dependent band-pass filter, as illustrated by the sound power measured by the microphone on Figure $2 \mathrm{~b}$ ). By analyzing the sound power spectrum for each angle of arrival and processing the spectral centroid for each angular orientation, one can map the directivity of the antenna as a function of frequency (Figure 2c)), thus allowing its use as a SMDF.

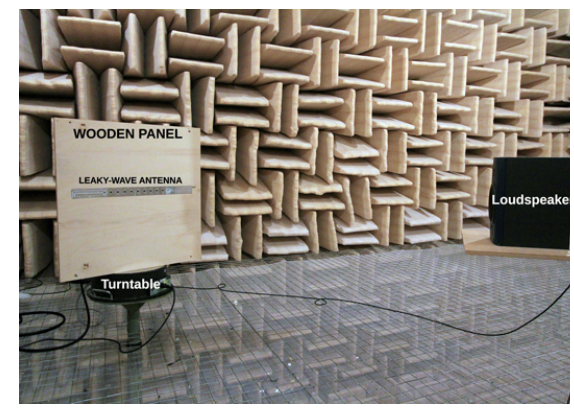

a)

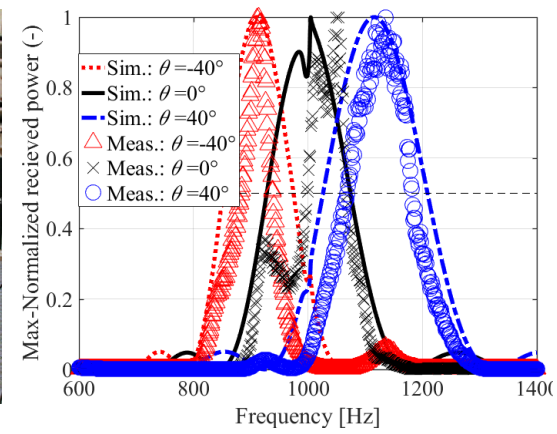

b)

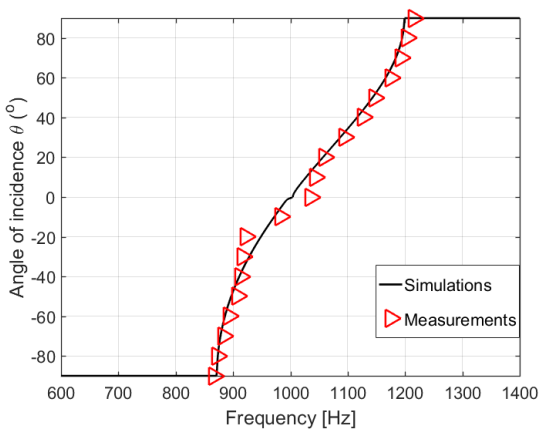

c)

Fig. 2: a) Experimental setup; b) Sound power spectrum measured by the microphone for different $\theta$ (compared to simulations); c) Measured directivity as a function of frequency, compared to simulations.

\section{CONCLUSiON}

The LWA structures proposed in this paper allows its application to both sound emission with frequencydependant directivity (ADP) and to the detection of the direction of sound arrival with a single microphone (SMDF). Although such properties can be achieved with conventional sum and delay beamforming techniques, this first design show the possibility to achieve such wave processing (as opposed to signal processing) with physical components only, and is likely to inspire future applications to the real world.

\section{ACKNOWLEDGEMENT}

This research was supported by the Swiss National Science Foundation under grant agreement 200020-138086/1.

\section{REFERENCES}

[1] V.G. Veselago "The electrodynamics of substances with simultaneously negative values of $\epsilon$ and $\mu$," Soviet Physics Uspekhi, , vol. 10(4), pp. 509-514, 1968.

[2] J. Li and C.T. Chen, "Double-negative acoustic metamaterial," Physical Review E, vol. 70(4), 055602, 2004.

[3] F. Bongard, H. Lissek and J.R. Mosig, "Acoustic transmission line metamaterial with negative/zero/positive refractive index," Physical Review B, vol. 82, 094306, 2010.

[4] H. Esfahlani, S. Karkar, J.R. Mosig and H. Lissek, "Acoustic Leaky-Wave antenna " Proc. of the 8th International Congress on Advanced Electromagnetic Materials in Microwaves and Optics, pp. 403-405, 2014.

[5] H. Esfahlani, S. Karkar, J.R. Mosig and H. Lissek, "Acoustic Dispersive Prism," Scientific Reports, vol. 6, 18911, 2016.

[6] H. Esfahlani, S. Karkar, J.R. Mosig and H. Lissek, "Exploiting the leaky-wave properties of transmission-line metamaterials for single-microphone direction finding," Journal of the Acoustical Society of America, vol. 139(6), pp. 3259-3266, 2016. 